\title{
RICHARD D. BRAUER
}

\author{
BY WALTER FEIT ${ }^{1}$
}

Richard Dagobert Brauer died on April 17, 1977 in Boston, Massachusetts at the age of 76. His death, after a short illness, was unexpected and came as a shock to his friends. During the past decade he had had several serious ailments but he had always recovered satisfactorily. There was no reason to believe that he would not recover from this final illness. He was mentally alert and mathematically active to the end, and this has made it all the more difficult to realize that he is now gone.

Brauer was born in Berlin on February 10, 1901. He was the youngest of three children of Max and Lilly Caroline Brauer. He showed an early interest in science and mathematics which was stimulated by his brother Alfred, who was older by seven years.

He graduated from high school in September, 1918. After graduation he and his classmates were drafted for civilian service in Berlin. As the first World War ended two months later, his war time service was brief and did not seriously interrupt his education. In contrast, his older brother Alfred spent four years in the German Army during the war and was seriously wounded.

In February 1919 he began his college education at the Technical University of Berlin. He soon realized that his interests were more theoretical than practical, and after one term he transferred to the University of Berlin. With the exception of a term at the University of Freiburg, he stayed at the University of Berlin until he was awarded his Ph.D. summa cum laude on March 16, 1926.

It was a custom at that time for students at one German University to spend some time at another. Brauer decided to spend a term at Freiburg. During that term he took a course on invariant theory with $\mathrm{O}$. Bolza. He also met Alfred Loewy, who was one of the professors at Freiburg, and with whom he was to continue a correspondence for many years.

During the time that Brauer was a student at Berlin, the University had a brilliant faculty in both mathematics and physics. He attended lectures by

AMS (MOS) subject classifications (1970). Primary 01A07.

${ }^{1}$ In preparing this article I have communicated with many friends of Richard Brauer and I wish to express my thanks to all of them. I especially wish to thank J. A. Green who has shown me a copy of the biographical part of the article which he is writing for the Bulletin of the London Mathematical Society. P. Fong and W. Wong have kindly allowed me to publish the bibliography which they have edited for Brauer's collected works.

Above all I wish to thank Mrs. Ilse Brauer who has at all times been willing to answer all my questions and supply information. Without her help much of this article could not have been written. 
Bieberbach, Carathéodory, Einstein, Knopp, von Laue, von Mises, Planck, E. Schmidt, I. Schur, Szegő and others. In his first term he took a course with Erhard Schmidt. Apparently Schmidt was not always well prepared. He frequently got stuck and improvised proofs on the spot. It was perhaps the first time that Brauer had seen a mathematician think about mathematics. This course left a lasting impression on him. Over fifty years later he recalled it fondly in the charming article [0] he wrote which is to serve as a preface to his collected works.

Among the faculty at Berlin it was however Issai Schur who had the most significant influence on Brauer's education. Schur taught courses and held seminars in number theory and algebra. In 1924 Schur wrote three papers on the representations of the real orthogonal group [Sc, vol. I, pp. 440-494]. In his thesis [1], which was written under Schur's guidance, Brauer gave a more algebraic approach to the subject and settled a question which had been left open by Schur. This question was answered independently by Hermann Weyl shortly thereafter in the more general context of the theory of representations of semisimple Lie groups. Aside from his thesis, Brauer's first published mathematical research was a joint paper with his brother and Heinz Hopf [2], both of whom were also students in Berlin at the time. This paper contains the solution to a problem which Schur had raised in his seminar.

One of Schur's courses affected Brauer's life in a vital but unexpected way. In November 1920, in Schur's course on Number Theory he met a fellow student named Ilse Karger, who was to become his wife on September 17, 1925. She was the daughter of a physician and took her Ph.D. in experimental physics in 1924. Shortly thereafter she realized that she was more interested in mathematics and took further courses in that subject with the idea of becoming a teacher. Over the years she has at various times held instructorships in mathematics at the University of Toronto and at Brandeis University. She was also an assistant professor at Boston University.

In 1975 Richard and Ilse Brauer celebrated their golden wedding anniversary. Their two sons George Ulrich, born in 1927 and Fred Günther, born in 1932, are both active research mathematicians.

Brauer's first academic position was an assistantship at the University of Königsberg. In 1927 he was promoted to Privatdozent and so obtained the right to give lectures. The department of mathematics was very small. There were two professors, K. Reidemeister and G. Szegő, W. Rogosinski and T. Kaluza were Privatdozents with the title of professor. As far as possible, these four together with Brauer had to cover the whole of mathematics in their lectures. Brauer was at Königsberg until 1933. During his years there he taught most of the usual courses but did not get a chance to lecture in the area of his mathematical research.

In his years at Königsberg his mathematical interests were centered on the theory of representations of groups and also on the structure of algebras. The intimate connection between these two subjects had only recently been recognized. This was at least partly due to his joint paper with E. Noether [4]. In this paper they exhibited the close relationship between splitting fields and 
maximal subfields of a simple algebra. Several other of his early papers are also concerned with questions concerning splitting fields of group representations and simple algebras.

In two very significant papers [12] and [13] he introduced what is now known as the Brauer group of a field. Let $F$ be a field. Consider the set of all isomorphism classes of central simple algebras over $F$. Define the product of two such algebras by the tensor product over $F$ and define two such algebras to be equivalent if they have isomorphic division ring components. The set of all equivalence classes forms the Brauer group $B(F)$ of $F$. In these papers the group $B(F)$ was studied by making use of what are now known as Brauer factor sets. These were first introduced in [3] and investigated further in [7]. He furthermore showed that a great deal of information concerning the structure of central division algebras over $F$ can be obtained from the study of $B(F)$ and its relationship to the group $B(K)$ as $K$ ranges over extension fields of $F$.

It is now recognized that $B(F)$ is an essential invariant of the field $F$ which has an importance independent of its usefulness in the study of central division algebras over $\boldsymbol{F}$. It has provided motivation for the development of the cohomology theory of groups and for the formulation of class field theory in cohomological terms.

The main problem in the theory of simple algebras is to describe all the division algebras with a given center $F$, or more modestly, to describe $B(F)$. If $F$ is algebraically closed then trivially $B(F)=1$. Already in 1878 Frobenius had shown that $B(R)$ is of order 2 if $R$ is the field of real numbers, or equivalently, the system of the Hamiltonian quaternions is the only central division algebra over $R$. [F, vol. I, p. 403]. In 1905 Wedderburn had shown that $B(F)=1$ in case $F$ is finite. By the twenties attention had begun to focus on the much deeper question of describing central simple algebras over algebraic number fields.

A central simple algebra $A$ over a field $F$ is cyclic if $A$ contains a subfield $K$ with $[A: F]=[K: F]^{2}$ such that $K$ is a Galois extension of $F$ with a cyclic Galois group. This concept was first introduced by L. E. Dickson. In his book [Di] published in 1923 he conjectured that every central simple algebra over an algebraic number field is cyclic. Dickson's book attracted much attention and was translated into German in 1927. In [12] Brauer gave the first example of a field $F$ and a central division algebra over $F$ which is not cyclic.

In 1931, in a joint paper by Brauer, H. Hasse and E. Noether [14], Dickson's conjecture was proved. This result is truly a "Hauptsatz". The paper contains not only a proof of the conjecture but also the description of a complete set of numerical invariants which characterize a central division algebra over a number field. This theorem is the climax of a long development in the theory of algebras which began with the work of Wedderburn in the U.S.A. and Schur in Germany before the first world war. Much of the structure theory of algebras which is needed for this paper had been developed independently and simultaneously by A. A. Albert, and the result is now justifiably known by the names of all four of these mathematicians. The proof is a triumph for the $p$-adic methods first introduced into number theory by $\mathrm{K}$. Hensel and then developed by $\mathrm{H}$. Hasse and depends on Hasse's prior 
classification of central simple algebras over a $p$-adic number field [H]. It is a striking example of a situation where the Hasse principle applies. (Actually the proof was not quite complete since it used the Grünwald existence theorem, and almost twenty years later S. Wang found that Grünwald's formulation was not quite correct [Wa1]. However Wang also showed that the error did not affect the application to the theory of algebras [Wa2].)

In $1898 \mathrm{H}$. Maschke [M] had shown that under certain assumptions the field of $n$th roots of unity is a splitting field for a group of order $n$. His work led to the natural conjecture that this is always the case. For solvable groups Schur had proved this in 1906 [Sc, vol. I, p. 194]. Somewhat surprisingly, the results of [14] are not strong enough to prove the conjecture in general. However in [14], as an application of the main results, Hasse showed that if $G$ is a group of order $n$ and $\chi$ is an absolutely irreducible character of $G$ then there exists an integer $h$, depending on $\chi$, such that the field of $\left(n^{h}\right)$ th roots of unity is a splitting field for $\chi$.

The Brauers found Königsberg a pleasant and comfortable place to live and to begin raising a family. The smallness of the mathematics department did not affect Brauer's work since he had made contacts in the larger world of German algebraists. Many years later he wrote in [96] "The intellectual atmosphere of German Universities of that period is remembered with nostalgia by all who knew it." His work before 1933, especially his contribution to the great paper [14], served to establish his reputation as an outstanding young mathematician.

Under normal circumstances he could confidently have looked forward to a Professorship at one of the major German Universities. However the circumstances were anything but normal. In 1933 Hitler became chancellor of Germany and soon thereafter he had established himself as dictator. Almost immediately the new German government began to implement its notorious antisemitic decrees. Very soon Brauer found himself without a position. Emmy Noether was treated the same way. Ironically, the third author of the famous paper on rational division algebras, $H$. Hasse, became a fervent supporter of the Nazis.

Brauer was fortunate in receiving an invitation to visit the University of Kentucky for the academic year 1933-1934. The money for his salary had been raised by the Emergency Committee for the aid of Displaced German Scholars with the help of the Jewish community in Lexington, Kentucky. He could read English but had not previously tried to speak the language. He embarked with a German-English dictionary and spent most of his time on the trip studying it. Nevertheless he arrived in Lexington speaking very little English. Ilse and the two boys had initially stayed behind in Berlin. They were able to join him three months later. (Brauer's brother Alfred was not dismissed immediately due to his war service. He stayed on in Germany until 1939 and then came to the United States. Their sister Alice stayed in Germany. She was killed in an extermination camp during the second world war.)

The Brauers received a warm welcome in Lexington, and some of his 
colleagues there became lifelong friends. Their introduction to the United States seems to have been as pleasant as it could be under the circumstances. The year in Lexington was a time of transition, during which all the members of the family were able to overcome their linguistic difficulties and to adapt very successfully to life in America.

In September, 1933 the Institute for Advanced Study opened in Princeton. Hermann Weyl was one of the original faculty. Brauer did not know Weyl personally but ever since he had written his thesis, he had hoped to meet him. At the International Congress in Bologna, he and Weyl had been present but he had been too shy to introduce himself or even to ask Szegö, who was there, to introduce him to Weyl. When he was invited to spend the academic year 1934-1935 at the Institute as Weyl's assistant it was, to paraphrase his own words in [0], "a fulfillment of a dream." This was one of those rather rare occasions when the reality lived up to expectations.

Brauer had an enormous admiration for Weyl which was evident whenever he referred to Weyl in later years. He once told me that he considered Weyl, more than anyone else, to be his teacher. In fact in [0] he wrote "I consider my years at the Institute in Princeton as the last part of my mathematical education." Clearly he didn't mean exactly that, since his mathematical education continued until the day of his death. I think he meant that this was the last time that he looked up to anyone as his teacher. Of course he was not exactly an ordinary pupil. While he benefitted greatly from his contact with Weyl, the benefits were not all one-sided. Weyl had a high regard for Brauer and in the preface to his book [We2], written in 1938, he acknowledged the importance of Brauer's work for the material presented in that book. The value of Brauer's work on the classical groups may thus be judged from the fact that Weyl's book has provided inspiration for several generations of mathematicians. Also in his treatment of generalized Riemann matrices [We1] he made use of Brauer factor sets, a concept he considered to be of fundamental importance.

During the year that Brauer was at the Institute, Weyl gave his famous lectures on the representations of semisimple groups. The notes were written up by Brauer. N. Jacobson had written up Weyl's notes on the classification of simple Lie algebras the previous year. With Weyl's encouragement Brauer found a proof that the Betti numbers of the classical groups were as conjectured by E. Cartan [21]. This result was obtained independently at about the same time by Pontrjagin by entirely different methods. Brauer and Weyl also wrote a joint paper on Spinors [19]. Some years later this paper was used by Kaufman and Onsager [K], [K-O] in their reformulation of Onsager's work on the Eising model which opened up the field for much further work. The papers [25], [26], and [29] appear to be later outgrowths of Weyl's influence. The results of [25] in particular were of great importance for Weyl's book [We2].

Other visitors in Princeton at the time included A. A. Albert, W. Magnus, C. L. Siegel and O. Zariski, as well as Jacobson mentioned above. All of them were to become lifelong friends with the Brauers. Emmy Noether who held a 
position at Bryn Mawr College came to Princeton every week to give a seminar. This was really the first time that the Brauers came to know her well on a personal level. They were deeply affected by her untimely death in the spring of 1935.

Another mathematician with whom Brauer became friendly at this time was Reinhold Baer. $\mathrm{He}$ and Brauer had actually grown up in the same neighborhood and had gone to the same school, but they had first met in 1931 when Brauer gave a talk at Halle where Baer was teaching at the time. However it was not until they had both come to the United States that they and their families became close.

In the fall of 1935 , at the age of 34 , he went to the University of Toronto as an assistant professor. Fortunately times have changed. Today it is hard to conceive of someone, already 34, with mathematical achievements on the level of Brauer's being offered, and accepting, an assistant professorship. $\mathrm{He}$ stayed in Toronto for thirteen years and when he left in 1948 he was a full professor and a fellow of the Canadian Royal Society. During his years at Toronto he did some of his most original mathematical research. He also turned out to be a very successful teacher. C. J. Nesbitt, who was his first $\mathrm{Ph}$.D. student was followed by many others. Those at Toronto included R. H. Bruck, S. A. Jennings, M. S. Mendelsohn, R. G. Stanton and R. Steinberg. Aside from those who received their Ph.D. under his guidance there are many more mathematicians (and some physicists) who were strongly influenced by him as graduate or undergraduate students.

Obviously Brauer's move to the United States marked a turning point in his life. It so happens that it also marked a turning point in the direction of his mathematical work. At any time, certain areas and problems in mathematics seem to be of central interest. In the twenties and early thirties this was the case with the theory of simple algebras and more particularly Dickson's conjecture. The proof of this conjecture in [14] ended an era. The theory of simple algebras became dormant for over a generation. A glance at Brauer's bibliography shows that after 1933 he wrote only three papers which were explicitly concerned with the structure of division algebras, namely [22], [30] and [50]. (Much later, in [67] and related papers he studied Schur indices of representations of finite groups. However his results here were obtained by entirely different methods which he had developed in the intervening two decades and will be discussed below.) The paper [50], which is a continuation of [15], is an attempt to apply the structure theory of simple algebras to the study of fields, rather than the other way around. In [30] he wrote down some results he had obtained on the question of whether a division algebra of index 5 is always cyclic. The question is still open. I would guess that these results had been proved much earlier and published at this time because his attention was on the subject, due to the fact that he had recently written [22].

The two unpublished papers [22] and [23] are explained by the following story. Originally they were solicited by Hasse (the second after the death of Emmy Noether) for the German encyclopedia of mathematics. A contract was signed and the papers were written and accepted. Shortly thereafter 
Brauer received a letter from the publisher informing him that they could not be published in Germany and sending him half the fee agreed on in the contract. This letter was accompanied by a legal document which quoted the appropriate German antisemitic law to justify this transaction. Brauer appealed to Hasse on the reasonable grounds that since he had done the work, he had earned the fee. However Hasse replied that it was impossible for him to do anything about it. This ended the matter.

It seems that Brauer hardly ever threw away any papers. After his death, the correspondence quoted above (with a copy of his own letter) and the papers [22] and [23] were found among his papers.

Brauer's first paper on the representations of finite groups over finite fields [18] appeared in 1935. The main result in this paper asserts that the number of absolutely irreducible representations of a finite group $G$ over a field of characteristic $p$ is equal to the number of $p^{\prime}$-classes of $G$. In this paper he also showed that an absolutely irreducible representation is determined by its trace function. According to [0], this paper was indirectly prompted by a suggestion of Schur. It was the precursor of his lifelong interest in what he called the theory of modular representations of finite groups. It should perhaps more accurately be called the theory of representations over local domains and their residue class fields. (J. P. Serre has pointed out that strictly speaking, [18] is a book not a paper. As such it is the only book which Brauer wrote and published. When he gave the Colloquium lectures to the American Mathematical Society in 1948 he had a manuscript for a book, but then unfortunately decided not to publish it.)

Brauer soon realized that before one could get at the deeper properties of modular representations of groups it was necessary to study the ring theoretic properties of group algebras. This work was begun in two joint papers with Nesbitt [27], [28]. These papers contain results about algebras which are then applied to group algebras. Questions concerning what are now called projective and injective modules are studied and the Cartan invariants are defined. In case of group algebras, the decomposition numbers are defined and the relations $\Sigma_{u} d_{u i} d_{u j}=c_{i j}$ connecting these two concepts are proved. The concept of a Brauer character (then called a modular character) first appeared in [27] and so did the concept of a block.

The paper [31] contains among other things the $p$-adic lifting of idempotents. This result was obtained independently by T. Nakayama who had also obtained some of the results of [27] and [28], for instance the Nakayama relations. Brauer and Nesbitt met Nakayama when he visited the United States on a fellowship. After Nesbitt received his Ph.D. and left Toronto, he and Nakayama in collaboration and individually continued to study rings and algebras from this point of view. Brauer was more interested in using the theory of algebras as a tool to study group representations. He did however publish some papers in ring theory in the next few years. In particular [36] contains some of his ideas in the theory of algebras and [41] contains an elementary proof of Hopkins' Theorem which asserts that in a left Artinian ring, a left ideal is either nilpotent or contains an idempotent. More in line with his main interests at the time was the joint paper with Nesbitt [34]. This paper recapitulates some of the necessary ring theory and contains the proofs 
of many of the basic results in the theory of modular representations. It also contains a description of all the irreducible modular representations of $S L_{2}\left(p^{n}\right)$. These latter results are the prototype for Steinberg's deep tensor product theorem which was proved about twenty years later in a paper that was very appropriately dedicated to Brauer on his sixtieth birthday [St]. The paper [34] remained the standard reference for anyone who wished to study modular representations for about twenty years.

While Brauer was developing the theory of modular representations, he was also looking for applications of the theory to questions concerning the structure of finite groups. In the short paper [32] he announced a large number of results whose proofs later appeared in [33], [37], [38], [39], [40] and [42]. The last three of these papers contain applications and the first three contain some necessary preliminaries. In [33] he showed that if $C$ is the Cartan matrix then det $C$ is a power of $p$. It is very easy to show that $C$ is nonsingular but the stronger result about $\operatorname{det} C$ is far from obvious. The method of proof was to lead to other important results which will be discussed below. In [37] he defined higher decomposition numbers and proved the orthogonality relations which they satisfy.

In my opinion [38] is one of the most remarkable and original papers that he ever wrote. This paper contains the description of ordinary and modular characters in a block of defect one and includes the definition of a tree, now known as the Brauer tree, associated to such a block. The results are too technical to be described here but they are quite unlike anything which had been known before about characters of finite groups. In cases when they are applicable they provide one of the most effective known methods for computing portions of a character table. These methods are especially useful if a Sylow group has prime order. Since it is quite rare for a simple group not to have at least one Sylow group of prime order, they are of wide applicability. At the time he discovered these results he tried to check them on known character tables. In one such case the results did not check and, as he told me many years later, he was rather unhappy for a while until he had shown that the error was in the character table and not in his results. After he had later defined the concept of a defect group it seemed plausible to expect that similar results should hold for blocks with a cyclic defect group. Brauer's methods could not be generalized and it was not until 1966 that John G. Thompson introduced a whole new approach, based on the work of J. A. Green [Gr1], [Gr2], which enabled E. C. Dade to extend these results to the case of a cyclic defect group [T2], [Da].

G. de B. Robinson, who was a colleague of Brauer's at Toronto, had conjectured that if $p$ is a prime and $G$ is a finite group which has a faithful complex representation of degree $d<(p-1) / 2$ then a Sylow $p$-group of $G$ is normal in $G$. In [39] and [40] Brauer proved the conjecture in case $p^{2}$ does not divide the order of $G$. Twenty years later John Thompson and I observed that a fairly simple argument reduced the general question to this case [FT1], so that Brauer had actually done most of the hard work. In the papers [39], [40] he also classified the groups in which equality can occur. To do this he 
used the full machinery that he had developed, including all the results in [38], to show that if $p>3$ then the group in question has a faithful 3 dimensional orthogonal representation over the field of $p$ elements. Since all subgroups of $O_{3}^{\prime}(p) \simeq P S L_{2}(p)$ are known, it follows easily that either the group has a normal Sylow $p$-group or, modulo its center, it is isomorphic to $\operatorname{PSL}_{2}(p)$.

Brauer once told me that one of his motives in studying modular representations was the hope of characterizing certain classical groups over finite fields. If for instance $G$ is supposed to be isomorphic to $G L_{n}(q)$ then one tries to show that $G$ has a faithful $n$ dimensional representation over the field of $q$ elements and so is isomorphic to a subgroup of $G L_{n}(q)$. Then presumably enough information should be available to show that $G$ is actually isomorphic to $G L_{n}(q)$. This was exactly the method used in [39] and [40]. However this method doesn't seem to work for any larger groups of Lie type. Even in [42], which contains a result related to those discussed above, the final recognition of the group in question comes from a theorem of Zassenhaus [Z] that characterizes certain permutation groups. It was soon realized by Brauer that the importance of modular representations lies in a different direction. Namely, the theory can be used to give new information about the character table of a group, which in turn can be applied to questions about the structure of the group.

In [47] he combined the modular representation theory with results from the theory of algebras and finally proved the conjecture that the field of $n$th roots of unity is a splitting field for a group of order $n$.

The three papers [43], [48], [49] contain announcements of further results in the theory of modular representations. Amongst other things the concept of a defect group is introduced and the First and Second Main Theorems of block theory are stated. Even the statements of these theorems, let alone their proofs, show great insight. There are no older results on which they could be modeled. They are a completely new departure. The proofs of the results announced in these papers did not appear until about ten years later in [65] and [73].

During the intervening decade Brauer worked in areas other than the theory of modular representations. This was at least partly due to his personal contact with E. Artin. He had met Artin briefly in Germany in 1928. It was not until a mathematical meeting in Chicago in the late thirties that they met again. At this meeting they became friendly and began to discuss mathematics. Brauer had a Guggenheim fellowship in 1941 and spent part of that year on a visit to Artin in Bloomington, Indiana. His contact with Artin aroused his interest in Artin's conjecture that Artin $L$-series (except for the Riemann zeta function) are entire functions. It was not even known at the time that they are meromorphic, however Artin pointed out to Brauer that this would follow if one could prove a certain result about characters of finite groups. Brauer had recently proved that the determinant of the Cartan matrix is a power of $p$ [33]. At some point in the next few years he realized that similar methods could be used to prove the required statement about group characters, and hence the fact that Artin $L$-series are meromorphic [51]. In a related paper [52] he shows that if $K$ is a Galois extension of the number field $F$ then 
$\zeta_{K} / \zeta_{F}$ is entire. This result had actually been obtained earlier by $\mathrm{H}$. Aramata [A]. An application of this result, also given in [52], was later strengthened in [58] where what is now known as the Brauer-Siegel theorem is proved. This gives asymptotic information about class numbers. In 1949 he was awarded the Cole prize of the American Mathematical Society for his work on Artin $L$-series.

Brauer's contact with Artin had important mathematical consequences. It was also very successful on a personal level. He and Artin became very friendly and so did their respective families. This friendship lasted until Artin's sudden death when Brauer had the sad duty of writing Artin's obituary [96].

By 1953 Brauer had recognized the unifying principle of much of his recent work. This is his famous theorem on the characterization of characters. An elementary group is a group of the form $A \times P$, where $A$ is cyclic and $P$ is a $p$-group. The following two results are proved in [62].

(I) Let $\theta$ be a class function defined on a group $G$ such that $\theta_{H}$ is a generalized character for every elementary subgroup $H$ of $G$. Then $\theta$ is a generalized character of $G$.

(II) Every generalized character of $G$ is of the form $\Sigma_{H} a_{H} \alpha_{H}^{G}$, where $H$ ranges over the elementary subgroups of $G$, each $\alpha_{H}$ is a generalized character of $H$ and each $a_{H}$ is an integer.

Statement (II) is implicit in [51]. It is explicit in [53], where it is used to simplify and strengthen the main result of [47] concerning splitting fields of finite groups. Further results about Schur indices derived from this point of view appear in [60]. The fact that the determinant of the Cartan matrix is a power of $p$ and related results are deduced from (I) in [62]. Of course the fact that Artin $L$-series are meromorphic is a consequence of (II).

The proofs in [62] are quite difficult. Roquette [R] gave a simpler proof and by incorporating some of his ideas into their own approach, Brauer and $\mathbf{J}$. Tate [63] were able to give a much more satisfactory proof. Since then, technical simplifications have been made but the general approach is that used in [63].

While in the midst of some of this work, Brauer left Toronto in 1948 for the University of Michigan at Ann Arbor. Nesbitt was on the faculty there but was in the process of moving into another area of mathematics. The other senior algebraist in the department was R. M. Thrall. Brauer was only at Michigan for four years, he accepted a professorship at Harvard in 1952. In this short time he had several Ph.D. students including K. A. Fowler, W. Jenner and D. J. Lewis. In addition to these W. P. Brown and J. P. Jans were at least partly his students, though they completed their dissertations after he had left. John Walter and I arrived at Michigan in 1951 and the courses we took with him during his last year there helped to shape our future mathematical interests.

He moved to Harvard in 1952 and stayed there until his retirement in 1970. His many Ph.D. students there included D. M. Bloom, P. Fong, M. E. Harris, I. M. Isaacs, H. S. Leonard, J. H. Lindsey, D. S. Passman, W. F. Reynolds, L. 
Solomon, D. B. Wales, H. N. Ward, and W. Wong. Life in the Boston area suited him both mathematically and socially. He and Ilse made many new friends and renewed old friendships. Their house in Belmont became familiar to many members of the large mathematical community in Boston, and also to visitors from other places. He and Ilse usually spent their summers in Pontresina, Switzerland with old friends such as C. L. Siegel. The rest of the time, even after his retirement, was spent in Boston except for visits to other universities.

At some time during the late forties Brauer had observed that some very simple properties of involutions (elements of order 2 in a group) can be used to derive some surprisingly strong results concerning the structure of groups of even order. In his thesis, Fowler used such results to give a characterization of the groups $S L_{2}\left(2^{n}\right)$ in terms of involutions. This thesis was never published. The paper [70], whose main result was proved independently by each of the three authors, includes the characterization of the groups $S L_{2}\left(2^{n}\right)$ as a special case.

In 1955 Brauer and Fowler published their joint paper [64]. This paper is remarkably simple and its main results can be understood by anyone who knows the definitions of a group. Yet the results and the point of view of this paper have turned out to be the key to much of the development of the theory of finite simple groups of even order since then. One of the results in [64] asserts that if $H$ is a finite group which contains an involution in its center then there are only a finite number of finite simple groups in which the centralizer of an involution is isomorphic to $H$. Thus it should be possible to classify these simple groups. The problem of classifying simple groups in terms of the structure of the centralizer of an involution has become known as Brauer's program. He explicitly proposed this in a talk he gave at the International Congress in Amsterdam in 1954 [68]. Among other results he announced the following in [68], though the proof did not appear until 1966, when a more general result was proved in [90].

THEOREM. Suppose that the finite group $G$ satisfies the following conditions.

(I) There exists an involution $J$ whose centralizer $\mathbf{C}(J)$ is isomorphic to $G L_{2}(q)$.

(II) If $C$ is an element of the center of $\mathbf{C}(J), C \neq 1$ then $\mathbf{C}(C)=\mathbf{C}(J)$.

(III) $G=G^{\prime}$.

If $q \equiv-1(\bmod 4), q \neq 1(\bmod 3)$ then either $G$ is isomorphic to $\operatorname{PSL}_{3}(q)$ or $q=3$ and $G$ is isomorphic to the Mathieu group $M_{11}$.

This was the first characterization of simple groups, other than $\operatorname{PSL}_{2}(q)$, in terms of involutions. Some geometric results, proved in [89] and [90], are required to recognize the group and the proof makes use of character theory, both ordinary and modular. The fact that there is an exceptional group was a harbinger of things to come. Several of the sporadic simple groups originally arose as exceptional cases in various characterization theorems.

A few years ago, as I was preparing a lecture on the history of group theory, I came across a paper of Burnside in which he characterized the 
groups $S L_{2}\left(2^{a}\right)$ for $a>1$ as the only simple groups of even order in which the order of every element is either 2 or odd [Bu1]. In this paper Burnside used some of the basic properties of involutions in a way quite similar to the way that Brauer used them fifty years later. However Burnside did not realize the importance of this approach. His paper had an innocuous title and appeared in a journal that is not readily available in mathematical libraries. I don't believe that he referred to the paper in his book [Bu2] and I don't know of any mathematical paper by Burnside or anyone else that refers to this paper. When I told Brauer about this paper, he was as surprised to hear of it as I had been when I first found it. It is a tribute to Brauer's insight that he realized that these extremely elementary arguments concerning involutions are of fundamental importance. The fact that a mathematician of the caliber of Burnside had overlooked the importance of such arguments, even after proving the result in [Bu1], shows that this insight was far from obvious.

The ten year delay in the publication of the proofs of the First and Second Main Theorem of block theory was only partly due to the fact that Brauer was working on other things. It was also partly due to the fact that he felt that no one was interested in his work on modular representations. This was not really the case. For instance I. M. Gelfand was interested in some of his work and quite recently he and some of his collaborators have proved results about the representations of Lie algebras which are analogous to some of Brauer's results in modular representation theory. See e.g. [BGG]. Of course Brauer could not know of Gelfand's interest. There were also some Japanese mathematicians who had become interested in the theory of modular representations. For instance M. Osima obtained alternative proofs for many of the basic results in the theory. He actually published the first proof of the First Main Theorem on blocks [Os]. This paper preceded Brauer's proof in [65] by about a year. Somewhat later, in a paper dedicated to Brauer's sixtieth birthday, H. Nagao gave an elegant proof of the Second Main Theorem on blocks [N]. Perhaps the first mathematician of the post war generation who mastered Brauer's work in group theory was M. Suzuki. He came to the United States in the early fifties and he has made many significant contributions to the theory of simple groups.

Aftern Brauer had proved his results about characters in blocks of defect one it was natural to study blocks with relatively simple defect groups. No general facts, or even conjectures, are known. However in special cases of "small" defect groups he was able to combine the results about involutions with the modular representation theory to get some results concerning the structure of simple groups. The following result, which was announced in a joint paper with M. Suzuki [74], must have convinced him of the importance of his methods.

THEOREM. If a Sylow 2-group of $G$ is a (generalized) quaternion group then $G$ is not simple.

There exist groups $G=G^{\prime}$ with quaternion Sylow 2-groups, for instance $G=S L_{2}(q)$ for odd $q$. Hence this result could never be proved by a transfer 
type argument. This was perhaps the first theorem since the work of Burnside and Frobenius half a century earlier which asserts that under suitable local hypotheses a group $G=G^{\prime}$ is not simple.

Shortly after the paper appeared it was realized that if the Sylow 2-group has order at least 16 then it is not necessary to use the theory of modular representations. Recently G. Glauberman [Gl] has succeeded in giving a proof without modular representations also in the case of a Sylow 2-group of order 8 .

Brauer wrote more than fifty papers after 1960. Most of them are in the theory of modular representations or its applications. I shall not attempt to describe this work but will make only a few comments.

The series of five papers [85], [86], [92], [112], [121] contain applications of block theory to questions concerning the structure of finite groups. Some of the most striking results are contained in the third one [92]. This contains, among other results, an elegant proof of the above mentioned theorem about groups with a quaternion Sylow 2-group, as well as the following result.

THEOREM. If a Sylow 2-group $S$ of $G$ is abelian on 2 generators and $G=G^{\prime}$ then $|S|=4$.

When J. G. Thompson published his thesis in 1959 [T1], it was immediately clear that the new direct methods he had introduced into the theory of finite groups were of fundamental importance. After Thompson and I had shown that groups of odd order are solvable [FT2], Brauer's work on involutions became even more central since it was now known to apply to all finite noncyclic simple groups. Many papers such as [77], [79], [91], [108], [110], [114], [115], [118] and [122] contain results which use modular representations, properties of involutions and, in some cases, the new methods which had originated with Thompson. Several of these papers were needed for the classification of all finite simple groups which do not contain an elementary abelian group of order 8. The proof of this result was completed in his joint paper with J. Alperin and D. Gorenstein [118].

The above mentioned theorems of Brauer are part of the work that is still going on, which aims at the classification of all finite simple groups. It now seems likely that this classification will be achieved in the near future (modulo some questions concerning groups of Ree type). When this is done it will be recognized that Brauer's ideas are an essential ingredient in such a classification.

For a long time Brauer had been intrigued by the work of H. F. Blichfeldt [B1]. In [98] he combined Blichfeldt's approach and his own methods to prove some theorems which include the classification of all finite groups with an irreducible 5 dimensional complex representation (Blichfeldt in [BI] had handled the groups in dimension at most 4). Later his students D. Wales and J. H. Lindsey used these methods to classify the finite groups with a faithful complex irreducible representation of dimension 7 and 6 respectively.

Many of his remaining papers explore various aspects of the theory of modular representations. For instance [100] and [102] contain many new 
results and include a proof of the Third Main Theorem on blocks. Some papers such as [105] contain alternative approaches to known results.

A mathematician is usually judged by several criteria. Among these are his ability to solve open problems of importance, his ability to introduce new concepts which clarify existing problems and his ability to develop coherent theories. In his work Brauer showed that he was a master of these three activities.

His work on the conjectures of Artin, Cartan and Dickson in [51], [21] and [14] respectively and his proof of the conjecture about splitting fields of finite groups in [47] are ample evidence of his ability to solve important open problems.

He introduced many new concepts and new points of view to the study of various questions in mathematics. A list of these would include the following.

The Brauer Group.

The definition and exploitation of generalized characters and the character ring, climaxed by his theorem on the characterization of characters.

The introduction of some basic arguments concerning involutions and the recognition of their importance.

The introduction of many concepts in the theory of modular representations. For instance: blocks, defect groups, Brauer characters, the Brauer correspondence.

For sheer originality I think that his work on modular representations is preeminent. He developed this theory and discovered and proved the three Main Theorems. He found unexpected and deep applications to group theory. Seldom in mathematics can one say that if a man hadn't done a piece of work it would not have been done for at least two or three decades. I think that this can be said of his work in the theory of modular representations.

I first met Brauer when I came to Michigan as Thrall's assistant after an undergraduate education very much in the Bourbaki tradition. I found Brauer's approach to mathematics less abstract. He was a mathematician in the tradition of Hermann Weyl. Although he collaborated with Emmy Noether, he was perhaps less influenced by her abstract point of view than any other German algebraist of his generation. This was a matter of temperament rather than training. He had his own way of doing and thinking about mathematics. For instance I took a reading course on class field theory with him. This meant that I read a set of his personal handwritten notes which consisted of a version of Chevalley's papers, rewritten in the older notation of Hasse. (Incidentally he had a beautifully clear handwriting. This was perhaps not so uncommon in his generation as they were still taught penmanship.)

When I first met Brauer he was fifty and I was twenty. I considered him an old man at the time. Nevertheless it was always easy for a graduate student to talk mathematics with him. This was due to the fact that he always had great enthusiasm for mathematics and he kept up in several fields. During the 
fifties he was abreast of everything that was going on in the theory of finite groups, he also knew of all the developments in class field theory and in the algebraic aspects of the theory of Lie groups. He was probably one of the first who understood Chevalley's Tôhoku paper. In general he read broadly and critically in the mathematical literature. For instance [45] and [57] are evidence of his breadth of interest. His memory was quite remarkable while I knew him. I wonder what it was like when he was twenty.

Many of his most important results have been given alternative and better proofs, usually by others, sometimes by himself. This is at least partly due to the fact that he was always primarily interested in results and did not spend a great deal of time looking for the "best" proof. However his papers are very readable. He had a style which enabled him to present complicated and computational proofs in an understandable form. He frequently wrote a paper and put it away for six months or a year before looking at it again. Then he either liked it or rewrote it. As any mathematician knows, things that are obvious in one's mind become a little less obvious a year later, so that his method is an excellent way to test readability.

Beginning with the late fifties, conferences on finite groups became fairly common and he attended many of them. He was frequently a generation older than most other participants. Suddenly there were a large number of young mathematicians who were familiar with his work. They treated him with respect not because of his age, but because of his achievements. I believe that he enjoyed such recognition from those who were competent to judge his work. In return he had a high regard for many of the younger mathematicians who were making significant progress in directions he had charted or in parallel directions. In [117] he had a rare opportunity to express such high esteem publicly. He was very pleased when the Nagoya Journal of Mathematics published an issue dedicated to him on his sixtieth birthday and equally pleased when the Journal of Algebra published an issue dedicated to him on his seventieth birthday.

After 1950 he began to receive mathematical recognition for his work on modular representations. He also received many honors. He was elected to the National Academy of Sciences in 1954. He was President of the American Mathematical Society in 1959-1960. In 1963 he was appointed an honorary member of the London Mathematical Society. In 1971 he was awarded the National Medal for Scientific Merit. He missed receiving the actual award from the President of the United States since he was in England as the Hardy lecturer in the spring of 1971.

He received honorary degrees from the University of Waterloo in 1968, the University of Chicago in 1969, the University of Notre Dame in 1974 and Brandeis University in 1975.

He was fortunate in that he lived long enough to receive the recognition which he deserved. He was even more fortunate in that his interest and abilities lasted to the end. He was a living counterexample to the myth that mathematics is only for the young. A glance at his bibliography shows that more than half his papers appeared after he was fifty.

I met him for the last time at a one day meeting in Storrs, Connecticut. I had been seriously ill and this was almost my first trip away from home in 
about five months. He gave a twenty minute talk (the paper appears in this issue of the Bulletin) and we spent a pleasant afternoon talking about mathematics and other things. As I was in my car about to leave for home, I saw him with Ilse and the Schafers come into the parking lot. It struck me that he was beginning to look his age both in his appearance and his walk. It was nevertheless an unexpected shock when I heard of his death six months later. In some ways he had already become part of mathematical history but in his constant mathematical activity he was very much part of the contemporary scene. His place among the important mathematicians of the twentieth century is secure, but those of us who knew him will miss him as a person.

\section{Bibliography OF RichaRd BRAUER}

0. Preface to his collected works written in 1976.

1. Über die Darstellung der Drehungsgruppe durch Gruppen linearer Substitutionen, InauguralDissertation zur Erlangung der Doktorwürde, Friedrich-Wilhelms-Universität, Berlin, 71 pp.

2. (with A. Brauer and $\mathbf{H}$. Hopf) Uber die Irreduzibilität einiger spezieller Klassen von Polynomen, Jber. Deutsch. Math.-Verein. 35 (1926), 99-112.

3. Uber Zusammenhänge zwischen arithmetischen und invariantentheoretischen Eigenschaften von Gruppen linearer Substitutionen, Sitzber. Preuss. Akad. Wiss. 1926, 410-416.

4. (with E. Noether), Über minimale Zerfällungskörper irreduzibler Darstellungen, Sitzber. Preuss. Akad. Wiss. 1927, 221-228.

5. Untersuchungen über die arithmetischen Eigenschaften von Gruppen linearer Substitutionen. I, Math. Z. 28 (1928), 677-696.

6. Über einen Satz für unitäre Matrizen, Tôhoku Math. J. 30 (1928), 72.

7. Uber Systeme hyperkomplexer Zahlen, Math. Z. 30 (1929), 79-107.

8. Die stetigen Darstellungen der komplexen orthogonalen Gruppe, Sitzber. Preuss. Akad. Wiss. 1929, 626-638.

9. (with A. Loewy), Über einen Satz für unitäre Matrizen, Tôhoku Math. J. 32 (1930), 44-49.

10. Uber Systeme hyperkomplexer Grossen, Jber. Deutsch. Math.-Verein. 38 (1929), 47-48.

11. (with I. Schur), Zum Irreduzibilitätsbegriff in der Theorie der Gruppen linearer homogener Substitutionen, Sitzber. Preuss. Akad. Wiss. 1930, 209-226.

12. Untersuchungen über die arithmetischen Eigenschaften von Gruppen linearer Substitutionen. II. Math. Z. 31 (1930), 733-747.

13. Uber die algebraische Struktur von Schiefkörpern, J. Reine Angew. Math. 166 (1932), 241-252.

14. (with H. Hasse and E. Noether), Beweis eines Hauptsatzes in der Theorie der Algebren, J. Reine Angew. Math. 167 (1931), 399-404.

15. Über die Konstruktion der Schiefkörper, die von endlichem Rang in bezug auf ein gegebenes Zentrum sind, J. Reine Angew. Math. 168 (1932), 44-64.

16. Über den Index und den Exponenten von Divisionalgebren, Tôhoku Math. J. 37 (1933), $77-87$.

17. Über die Kleinsche Theorie der algebraischen Gleichungen, Math. Ann. 110 (1934), 473-500.

18. Uber die Darstellung von Gruppen in Galoisschen Feldern, Actualités Sci. Indust., no. 195, Hermann \& Cie, Paris, 1935.

19. (with H. Weyl), Spinors in $n$ dimensions, Amer. J. Math. 57 (1935), 425-449.

20. (with A. Brauer), Über Irreduzibilitätskriterien von I. Schur und G. Pólya, Math. Z. 40 (1935), 242-265.

21. Sur les invariants intégraux des variétés représentatives des groupes de Lie simples clos, C. $\mathbf{R}$. Acad. Sci. Paris 201 (1935), 419-421. 
22. Algebra der hyperkomplexen Zahlensystem (Algebren), Accepted for publication (1936) in Enzyklöpadie der Mathematischen Wissenschaften vol. IB 8, B. G. Teubner, Leipzig. About 50 pp. ${ }^{*}$

23. Symmetrische Funktionen. Invarianten von linearer Gruppen endlicher Ordnung, Accepted for publication (1936) in Enzyklopädie der Mathematischen Wissenschaften vol. I B12, B. G. Teubner, Leipzig. About 50 pp."

24. A characterization of null systems in projective space, Bull. Amer. Math. Soc. 42 (1936), 247-254.

25. On algebras which are connected with the semisimple continuous groups, Ann. of Math. 38 (1937), 857-872.

26. Eine Bedingung für vollständige Reduzibilität von Darstellungen gewöhnlicher und infinitesimaler Gruppen, Math. Z. 41 (1936), 330-339

27. (with C. Nesbitt), On the modular representations of groups of finite order. I, Univ. Toronto Studies no. 4 (1937), 21 pp.

28. (with C. Nesbitt), On the regular representations of algebras, Proc. Nat. Acad. Sci. U.S.A. 23 (1937), 236-240.

29. Sur la multiplication des caractéristiques des groupes continus et semi-simples, C. R. Acad. Sci. Paris 204 (1937), 1784-1786.

30. On normal division algebras of index 5, Proc. Nat. Acad. Sci. U.S.A. 24 (1938), 243-246.

31. On modular and p-adic representations of algebras, Proc. Nat. Acad. Sci. U.S.A. 25 (1939), 252-258.

32. On the representation of groups of finite order, Proc. Nat. Acad. Sci. U.S.A. 25 (1939), 290-295.

33. On the Cartan invariants of groups of finite order, Ann. of Math. 42 (1941), 53-61.

34. (with C. Nesbitt), On the modular characters of groups, Ann. of Math. 42 (1941), 556-590.

35. (with H. S. M. Coxeter), A generalization of theorems of Schönhardt and Mehmke on polytopes, Trans. Roy. Soc. Canada Sect. III (3) 34 (1940), 29-34.

36. On sets of matrices with coefficients in a division ring, Trans. Amer. Math. Soc. 49 (1941), $502-548$.

37. On the connection between the ordinary and the modular characters of groups of finite order, Ann. of Math. 42 (1941), 926-935.

38. Investigations on group characters, Ann. of Math. 42 (1941), 936-958.

39. On groups whose order contains a prime number to the first power. I, Amer. J. Math. 64 (1942), 401-420.

40. On groups whase order contains a prime number to the first power. II, Amer. J. Math. 64 (1942), $421-440$.

41. On the nilpotency of the radical of a ring, Bull. Amer. Math. Soc. 48 (1942), 752-758.

42. On permutation groups of prime degree and related classes of groups, Ann. of Math. 44 (1943), 57-79.

43. On the arithmetic in a group ring, Proc. Nat. Acad. Sci. U.S.A. 30 (1944), 109-114.

44. On hypercomplex arithmetic and a theorem of Speiser, Festschrift for 60th birthday of Andreas Speiser, Zürich, 1945, 13 pp.

45. A note on systems of homogeneous algebraic equations, Bull. Amer. Math. Soc. 51 (1945), 749-755.

46. (with H. F. Tuan), On simple groups of finite order, Bull. Amer. Math. Soc. 51 (1945), 756-766.

47. On the representation of a group of order $g$ in the field of the $g$ th roots of unity, Amer. J. Math. 67 (1945), 461-471.

48. On blocks of characters of groups of finite order. I, Proc. Nat. Acad. Sci. U.S.A. 32 (1946), 182-186.

49. On blocks of characters of groups of finite order. II, Proc. Nat. Acad. Sci. U.S.A. 32 (1946),

215-219.

50. On splitting fields of simple algebras, Ann. of Math. 48 (1947), 79-90.

51. On Artin's L-series with general group characters, Ann. of Math. 48 (1947), 502-514.

52. On the zeta-functions of algebraic number fields, Amer. J. Math. 69 (1947), 243-250.

\footnotetext{
\# Not published because of political considerations.
} 
53. Applications of induced characters, Amer. J. Math. 69 (1947), 709-716.

54. On a conjecture by Nakayama, Trans. Roy. Soc. Canada Sect. III (3) 41 (1947), 11-19.

55. A note on Hilbert's Nullstellensatz, Bull. Amer. Math. Soc. 54 (1948), 894-896.

56. Representations of groups and rings, Amer. Math. Soc. Colloquium lectures, 1948, 22 pp.

57. On a theorem of H. Cartan, Bull. Amer. Math. Soc. 55 (1949), 619-620.

58. On the zeta-functions of algebraic number fields. II, Amer. J. Math. 72 (1950), 739-746.

59. Beziehungen zwischen Klassenzahlen von Teilkörpern eines galoisschen Körpers, Math. Nachr. 4 (1951), 158-174.

60. On the algebraic structure of group rings, J. Math. Soc. Japan 3 (1951), 237-251.

61. On the representations of groups of finite order, Proc. Internat. Congr. Math. (Cambridge, Mass., 1950), Vol. 2, Amer. Math. Soc., Providence, R. I., 1952, pp. 33-36.

62. A characterization of the characters of groups of finite order, Ann. of Math. 57 (1953), 357-377.

63. (with J. Tate), On the characters of finite groups, Ann. of Math. 62 (1955), 1-7.

64. (with K. A. Fowler), On groups of even order, Ann. of Math. 62 (1955), 565-583.

65. Zur Darstellungstheorie der Gruppen endlicher Ordnung, Math. Z. 63 (1956), 406-444.

66. (with N. C. Ankeny and S. Chowla), A note on the class-numbers of algebraic number fields, Amer. J. Math. 78 (1956), 51-61.

67. Number theoretical investigations on groups of finite order, Proc. Internat. Sympos. on Algebraic Number Theory, Tokyo-Nikko, 1955, pp. 55-62.

68. On the structure of groups of finite order, Proc. Internat. Congr. Math., Amsterdam, 1954,

Vol. 1, Noordhoff, Groningen; North-Holland, Amsterdam, 1957, pp. 209-217.

69. Some remarks on associative rings and algebras, Nat. Acad. Sci.-Nat. Res. Council, Publ. 502, Washington, 1957.

70. (with M. Suzuki and G. E. Wall), A characterization of the one-dimensional unimodular projective groups over finite fields, Illinois J. Math. 2 (1958), 718-745.

71. (with W. F. Reynolds), On a problem of E. Artin, Ann. of Math. 68 (1958), 713-720.

72. (with W. Feit), On the number of irreducible characters of finite groups in a given block, Proc.

Nat. Acad. Sci. U.S.A. 45 (1959), 361-365.

73. Zur Darstellungstheorie der Gruppen endlicher Ordnung. II, Math. Z. 72 (1959), 25-46.

74. (with M. Suzuki), On finite groups of even order whose 2-Sylow group is a quaternion group, Proc. Nat. Acad. Sci. U.S.A. 45 (1959), 1757-1759.

75. Les groupes d'ordre fini et leurs caractères, Sém. P. Dubreil, M. L. Dubreil-Jacotin et C. Pisot, Paris, 1959, 6-01 to 6-16.

76. On blocks of representations of finite groups, Proc. Nat. Acad. Sci. U.S.A. 47 (1961), 1888-1890.

77. Investigation on groups of even order. I, Proc. Nat. Acad. Sci. U.S.A. 47 (1961), 1891-1893.

78. (with H. S. Leonard), On finite groups with an abelian Sylow group, Canad. J. Math. 14 (1962), 436-450.

79. On groups of even order with an abelian 2-Sylow subgroup, Arch. Math. 13 (1962), 55-60.

80. On some conjectures concerning finite simple groups, Studies in Math. Analysis and Related Topics, Stanford, 1962, pp. 56-61.

81. On finite groups and their characters, Bull. Amer. Math. Soc. 69 (1963), 125-130.

82. Representations of finite groups, Lectures on Modern Math. vol I, Wiley, New York, 1963, pp. 133-175.

83. On quotient groups of finite groups, Math. Z. 83 (1964), 72-84.

84. A note on theorems of Burnside and Blichfeldt, Proc. Amer. Math. Soc. 15 (1964), 31-34.

85. Some applications of the theory of blocks of characters of finite groups. I, J. Algebra 1 (1964), 152-167.

86. Some applications of the theory of blocks of characters of finite groups. II, J. Algebra 1 (1964), 307-334.

87. On certain classes of positive definite quadratic forms, Acta Arith. 9 (1964), 357-364.

88. On the relation between the orthogonal group and the unimodular group, Arch. Rational Mech. Anal. 18 (1965), 97-99.

89. On finite desarguesian planes. I, Math. Z. 90 (1965), 117-123.

90. On finite desarguesian planes. II, Math. Z. 91 (1966), 124-151.

91. Investigation on groups of even order. II, Proc. Nat. Acad. Sci. U.S.A. 55 (1966), 254-259. 
92. Some applications of the theory of blocks of characters of finite groups. III, J. Algebra 3 (1966), 225-255.

93. (with P. Fong), A characterization of the Mathieu group $M_{12}$, Trans. Amer. Math. Soc. 122 (1966), 18-47.

94. (with W. Feit), An analogue of Jordan's theorem in characteristic p, Ann. of Math. 84 (1966), 119-131.

95. Some results on finite groups whose order contains a prime to the first power, Nagoya Math. J. 27 (1966), 381-399.

96. Emil Artin, Bull. Amer. Math. Soc. 73 (1967), 27-43.

97. On simple groups of order $5 \cdot 3^{a} \cdot 2^{b}$, Mimeographed notes, Harvard Univ., 1967, $49 \mathrm{pp}$.

98. Uber endliche lineare Gruppen von Primzahlgrad, Math. Ann. 169 (1967), 73-96.

99. On a theorem of Burnside, Illinois J. Math. 11 (1967), 349-352.

100. On blocks and sections in finite groups. I, Amer. J. Math. 89 (1967), 1115-1136.

101. On pseudo groups, J. Math. Soc. Japan 20 (1968), 13-22.

102. On blocks and sections in finite groups. II, Amer. J. Math. 90 (1968), 895-925.

103. On simple groups of order $5 \cdot 3^{a} \cdot 2^{b}$, Bull. Amer. Math. Soc. 74 (1968), 900-903.

104. On a theorem of Frobenius, Amer. Math. Monthly 76 (1969), $12-15$.

105. Defect groups in the theory of representations of finite groups, Illinois J. Math. 13 (1969), 53-73.

106. On the order of finite projective groups in a given dimension, Nachr. Akad. Wiss. Göttingen Math.-Phys. Kl. II 1969, 103-106.

107. On the representations of finite groups, Yeshiva Univ., 1969, 121-128.

108. On groups with quasi-dihedral Sylow 2-subgroups. II, Theory of Finite Groups, a symposium, Benjamin, 1969, pp. 13-19.

109. On the first main theorem on blocks of characters of finite groups, Illinois J. Math. 14 (1970), 183-187.

110. (with J. L. Alperin and D. Gorenstein), Finite groups with quasi-dihedral and wreathed Sylow 2-subgroups, Trans. Amer. Math. Soc. 151 (1970), 1-261.

111. On finite desarguesian planes. III, Math. Z. 117 (1970), 76-82.

112. Some applications of the theory of blocks of characters of finite groups. IV, J. Algebra 17 (1971), 489-521.

113. Types of blocks of representations of finite groups, Proc. Sympos. Pure Math., vol. 21, Amer. Math. Soc., Providence, R. I., 1971, pp. 7-11.

114. (with W. J. Wong), Some properties of finite groups with wreathed Sylow 2-subgroup, J. Algebra 19 (1971), 263-273.

115. Character theory of finite groups with wreathed Sylow 2-subgroups, J. Algebra 19 (1971), 547-592.

116. Blocks of characters, Proc. Internat. Congr. Math. 1970, vol. 1, 341-345.

117. On the work of John Thompson, Proc. Internat. Congr. Math. 1970, vol. 1, 15-16.

118. (with J. L. Alperin and D. Gorenstein), Finite simple groups of 2-rank two, Scripta Math. 29 (1973), 191-214.

119. A note on zeta-functions of algebraic number fields, Acta Arith. 24 (1973), 325-327.

120. On the structure of blocks of characters of finite groups, Proc. Second Internat. Conf. Theory of Groups (Canberra, 1973), 103-130.

121. Some applications of the theory of blocks of characters of finite groups. V, J. Algebra 28 (1974), 433-460.

122. On 2-blocks with dihedral defect groups, Symposia Math. (INDAM) 13 (1974), 367-393.

123. (with P. Fong), On the centralizers of p-elements in finite groups, Bull. London Math. Soc. 6 (1974), 319-324.

124. On the resolvent problem, Ann. Mat. Pura Appl. 102 (1975), 45-55.

125. On finite groups with cyclic Sylow subgroups. I, J. Algebra 40 (1976), 556-584.

126. Notes on representations of finite groups. I, J. London Math. Soc. (2) 13 (1976), 162-166.

127. Blocks of characters and structure of finite groups, Bull. Amer. Math. Soc. 85 (1979),

128. On finite projective groups, Contributions to algebra, Academic Press, New York, 1977, pp. 63-82. 


\section{REFERENCES}

[A] H. Aramata, Über die Teilbarkeit der Dedekindschen Zetafunktionen, Proceedings of the Imperial Acad. of Japan 9 (1933), 31-34.

[BGG] I. N. Bernstein, I. M. Gelfand and S. I. Gelfand, Category of g modules, Functional Analysis and its Applications 10 (1976), 87-92.

[BI] H. F. Blichfeldt, Finite collineation groups, Univ. of Chicago Press, Chicago, IIl., 1917.

[Bu1] W. Burnside, On a class of groups of finite order, Transactions of the Cambridge Philos. Soc. 18 (1900), 269-276.

[Bu2] _ , Theory of groups of finite order 2 nd ed., Cambridge Univ. Press, London and New York, 1911.

[Da] E. C. Dade, Blocks with cyclic defect groups, Ann. of Math. (2) 84 (1966), $20-48$.

[Di] L. E. Dickson, Algebras and their arithmetics, Univ. of Chicago Press, Chicago, Ill., 1923.

[FT1] W. Feit and J. G. Thompson, Groups which have a faithful representation of degree less than $(p-1) / 2$, Pacific J. Math. 11 (1961), 1257-1262.

[FT2] __ Solvability of groups of odd order, Pacific J. Math. 13 (1963), 755-1029.

[F] G. Frobenius, Collected works, Springer-Verlag, Berlin, Heidelberg, New York, 1968.

[GI] G. Glauberman, On groups with a quaternion Sylow 2-subgroup, Illinois J. Math. 18 (1974), 60-65.

[Gr1] J. A. Green, On the indecomposable representations of a finite group, Math. Z. 70 (1959), $430-445$.

[Gr2] —, Blocks of modular representations, Math. Z. 79 (1962), 100-115.

[H] H. Hasse, Über p-adische Schiefkörper und ibre Bedeutung für die Arithmetik hypercomplexer Zahlkörper, Math. Ann. 104 (1931), 495-534.

[K] B. Kaufman, Crystal statistics. II, Partition function evaluated by Spinor analysis, Phys. Rev. $76(1949), 1232-1243$.

[K-O] B. Kaufman and L. Onsager, Crystal statistics. III, Short range order in a binary Eising lattice, Phys. Rev. 76 (1949), 1244-1252.

[M] H. Maschke, Ueber den Arithmetischen Charakter der Coefficienten der Substitutionen endlicher linearer Substitutionsgruppen, Math. Ann. 50 (1898), 482-498.

[N] H. Nagao, A proof of Brawer's theorem on generalized decomposition numbers, Nagoya Math. J. 22 (1963), 73-77.

[Os] M. Osima, Notes on blocks of group characters, Math. J. Okayama Univ. 4 (1955), 175-188.

[R] P. Roquette, Arithmetische Untersuchung des Charakterringes einer endlichen gruppe, J.

Reine. Angew. Math. 190 (1952), 148-168.

[Sc] I. Schur, Collected works, Springer-Verlag, Berlin, Heidelberg, New York, 1973.

[St] R. Steinberg, Representations of algebraic groups, Nagoya Math. J. 22 (1963), 33-56.

[T1] J. G. Thompson, Normal p-complements for finite groups, Math. Z. 72 (1960), 332-354.

[T2] _ Vertices and sources, J. Algebra 6 (1967), 1-6.

[Wa1] S. Wang, A counter-example to Grunwald's theorem, Ann. of Math. (2) 49 (1948), 1008-1009.

[Wa2] __, On Grunwald's theorem, Ann. of Math. (2) 51 (1950), 471-484.

[We1] H. Weyl, Generalized Riemann matrices and factor sets, Ann. of Math. (2) 37 (1936), 709-745.

[We2] _ Classical groups, Princeton Univ. Press, Princeton, N. J., 1946.

[Z] H. Zassenhaus, Kennzeichnung endlicher linearer Gruppen als Permutationsgruppen, Abh. Math. Seminar, Hamburg Univ. 11 (1936), 17-40.

Department of Mathematics, Yale University, New Haven, Connecticut 06520 